\title{
Omnidirectional Locomotion in a Quadruped Robot: A CPG-based Approach
}

\author{
Vítor Matos, Cristina P. Santos
}

\begin{abstract}
Quadruped locomotion on rough terrain and unpredictable environments is still a challenge, where the concept of Central Pattern Generators (CPG) has brought interesting ideas.

In this contribution we present a CPG design based on coupled oscillators, generating the required stepping movements of a limb for omnidirectional motion. Movements are online modulated through small value changes in the CPG's parameters as required to perform the desired omnidirectional locomotion in a quadruped robot. We also present a methodology to modulate the CPG's parameters, reducing the control dimensionality, described in terms of the robot's translational speed, angular velocity and walking orientation.

Results show the proposed controller is well suited for the online generation and modulation of the motor patterns required to achieve the desired omnidirectional walking motion.
\end{abstract}

\section{INTRODUCTION}

The robot capability of walking to any point of interest and navigate in its environment is the addressed goal in this contribution. We explore an approach that uses dynamical systems to generate and control omnidirectional locomotion. Our approach is partly inspired from the biological concepts of Central Pattern Generators (CPGs) [1], [2], i.e. spinalneural networks capable of autonomously producing coordinate rhythmic output signals; and by the concepts of force fields [3].

This dynamical systems approach has proven to be successful in many robotic applications [4]-[10]. It offers multiple interesting features, including: low computational cost; the intrinsic stability properties allow for feedback integration; smooth trajectories modulated by simple parameters change; provide for coupling/synchronization; and entrainment phenomena.

The proposed model addresses the role of the spinal cord and generates the motor patterns by networks of CPGs. It is based on past work for modeling a limb-CPG [8], [9], [11]: a unique set of (oscillator-based) differential equations is able to produce complex movements modeled as periodic movements around time-varying offsets. We use Hopf oscillators that allow online modulation of the trajectories with respect to their amplitude, frequency, oscillator's couplings and to the offsets of the motor patterns by small parameter changes.

In this contribution, omnidirectional locomotion in a rigid bodied robot is achieved by a combined use of the flap and swing hip joints, based on the ideas of the wheel model [12].

Vítor Matos and Cristina Santos are with Industrial Electronics Department, School of Engineering, University of Minho, 4800058 Guimaraes, Portugal vmatos@dei.uminho.pt, cristina@dei.uminho.pt
The proposed network structure is well suited for this method since it allows to independently control the step movements of the different joints while still keeping the intralimb and interlimb coordination.

CPG parameters for hip swing and flap are then set as required to perform the desired omnidirectional locomotion. We present a method for determining the values of the parameters described merely in terms of the robots high level operational locomotion parameters, as the desired walking orientation, translational speed and angular velocity of the desired motion.

In the overall this is a step forward in our research towards adaptive locomotion for a quadruped robot over rough terrain. We have been progressively advancing, focusing on the open loop aspects of the locomotion generation, such as the required motor primitives [9]; gait transition [11]; postural control [8] and currently we are addressing the inclusion of sensory-feedback. Herein, we extend the ideas presented in [10] for a similar architecture towards the achievement of the omnidirectional locomotion behavior. Control of omnidirectional locomotion has not been fully explored but it is very relevant in terms of manoeuvrability.

Control approaches based on CPGs and nonlinear dynamical systems are widely used in robotics to achieve tasks which involve rhythmic motions such as biped and quadruped autonomous locomotion over irregular terrain [13], juggling [14], drumming [6], playing with a slinky toy and basis field approaches for limb movements [3].

Quadruped omnidirectional locomotion has been achieved through several methods. The most usual method for achieving omnidirectional motion is planning the footholds and gaits through the use of inverse kinematics and body dynamics [15]-[17]. Parameterizable omnidirectional walk can also be found in [12], where optimization was used to find the best parameters for the leg motions. Omnidirectional path following was successfully achieved in [18], by optimizing the parameters simultaneously for all directions of motion and turning rates.

There are a couple of implementations of CPG based controllers where quadruped steering is achieved, but not omnidirectional locomotion. Tsujita and colleagues [19] proposed a dynamic turning control system for a quadruped robot by using nonlinear oscillators. The suggested steering approach is not suitable for our robotic platform which has a rigid body. Hiroshi Kimura and colleagues [20] designed a locomotor controller based on neural systems. However, their steering approach is specific for a quadruped robot with yaw joints on the legs. 
We continue using the previous formulated framework [8], [9], [11], build upon Hopf oscillators which enable complete control over their control states, with a modular and functional organization.

We present results of the experiments performed on the AIBO robot. The obtained results demonstrate the adequacy of the proposed locomotor controller to generate the desired motion in terms of the walking velocity, orientation and angular velocity.

\section{DYNAMICAL SYSTEM}

Consider a nonlinear dynamical oscillator, containing a supercritical Hopf bifurcation, given by the following differential equations:

$$
\begin{aligned}
\dot{x} & =\alpha\left(\mu v-r^{2}\right)(x-O)-\omega \imath z \\
\dot{z} & =\alpha\left(\mu v-r^{2}\right) z+\omega \imath(x-O), \\
\omega & =\frac{\frac{1-\beta}{\beta} \omega_{\mathrm{sw}}}{\mathrm{e}^{-a z}+1}+\frac{\omega_{\mathrm{sw}}}{\mathrm{e}^{a z}+1}
\end{aligned}
$$

where $r=\sqrt{(x-O)^{2}+z^{2}}, x$ and $z$ are the state variables. The possible solutions for this dynamical system are an harmonic oscillation around $(x, z)=(O, 0)$ (for $\mu v>0$ ) with frequency $\omega$, or a stable fixed point at $(x, z)=(O, 0)$ (for $\mu v<0$ ), which speed of convergence is controlled by $\alpha$. The variable $O$ is used to control the $x$ solution offset. Parameter $v \in\{-1,1\}$ switches on/off the rhythmic activity. Amplitude of the oscillations is given by $\sqrt{\mu}$, for $v=1$. Hence, this oscillator is able to generate both:

1) a discrete movement to a time-varying offset $O$;

2) a rhythmic movement around an offset $O$;

3) and the superimposition of both.

These ideas were further explored in [7], [9], where this offset becomes the state variable of another dynamical system.

Parameter $l$ specifies the limit-cycle direction. The limitcycle rotates clockwise or counterclockwise if $l=-1$ or $\imath=1$, respectively.

Oscillations' frequency is specified by $\omega$. Eq. 3 alternates the value of $\omega$ between two different values, enabling to independently control the durations of the ascending and descending parts of the $x$ solution (see [10]) through the specification of the duty factor $\beta$.

The modulation of the generated trajectories is carried out explicitly through the specification of these parameters. The outcome of the changes result in straightforward and smooth modulation of the trajectories.

The integration of feedback mechanisms is possible and has been explored in other works [8]-[10] however is not addressed in this contribution.

\section{Central Pattern Generators}

The controller is based on the biological concept of central pattern generators (CPGs) [2], i.e. functional organizations in the animals' neurological system that control the bodies' rhythmic movements.

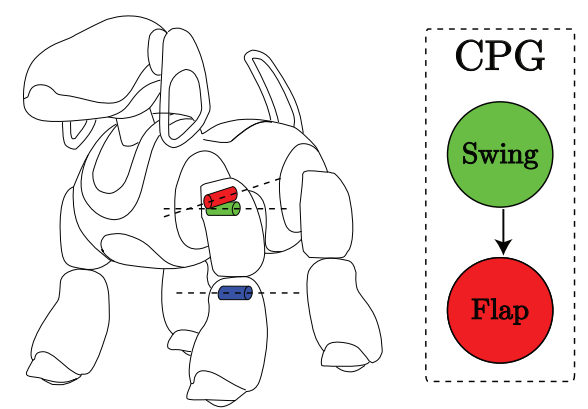

Fig. 1. The limb-CPG is composed by two unit-CPGs, each controlling a single hip joint.

The concept of biological locomotor CPG includes the idea of hierarchical organized unitary oscillators, the unitCPGs. A single unit-CPG controls and activates the antagonistic muscle pairs, controlling the movements of a single joint. Movements of a limb are controlled by a limb-CPG, composed by a group of coordinated unit-CPGs within a limb. In the end, a network of four coupled limb-CPGs constitutes the proposed locomotion controller.

\section{A. Unit-CPG}

A unit-CPG is modeled by the nonlinear Hopf oscillator described in section II. The generated $x$ solution is used as the control trajectory for a joint of the robot limbs. These trajectories encode the values of the joint's angles $\left(^{\circ}\right)$ and are sent online to hardware PID controllers of each limb joint.

The oscillator is able to generate motor patterns without any input activity and sensory feedback, when activated by simple commands that somehow encode their rhythmic activation, frequency, direction and amplitude. These features are similar to their biological counterparts.

We are able to control the duty factor by keeping the swing duration constant and changing only the stance duration, thus achieving different quadrupedal gaits.

The set of equations also allows an independent control of the limit-cycle direction, depending on the parameter $t$. Changes in the limit-cycle direction results in changing the step stance phase between descending $(l=1)$ and ascending $(l=-1)$ phases of the $x$ trajectory, allowing to choose between forward/backward stepping and left/right stepping.

Each unit-CPG takes a set of parameters for the modulation of the generated trajectories for the specified joint, $\{v, \mu, \imath, O, \beta\}$. These parameters do not need to be hand tuned and their values are specified by the mechanism presented in the next sections, controlling the parameters for omnidirectional locomotion. The parameters $\alpha, \omega_{\mathrm{sw}}$ and $a$ are set a priori.

\section{B. Limb-CPG}

The limb-CPG controls and coordinates the movements in a single limb. It is composed by a pair of unit-CPGs: one controlling the hip swing joint (subscript s) and the other controlling the hip flap joint (subscript f) (fig. 1).

In order to execute a step it is necessary to ensure a proper synergy of movements in all joints within a limb. It is thus 
required that these movements are expressed correctly, with coordinated step phases in both joints. We can therefore unilaterally couple the swing unit-CPG to the flap unit-CPG. This is achieved by changing the flap's $z$ differential equation (1) as follows,

$$
\dot{z}_{\mathrm{f}}=\ldots+\kappa \frac{z_{\mathrm{s}}}{r_{\mathrm{s}}}
$$

where $\kappa$ is the coupling strength. The unit-CPGs are coupled through $z$ because allows the simplest coupling method, where it is possible to independently control the directions of oscillations on both unit-CPGs.

On this work, the knee joints are controlled as simple as possible. Flexing and extending the knees to fixed angles, depending on step phase.

\section{CPG network}

In order to generate the adequate stepping sequence for each limb, the four limb-CPGs must be coordinated. We couple the four limb-CPGs, at the unit-CPG level, ensuring a correct coordination between the limbs. Unit-CPGs are coupled as follows

$$
\left[\begin{array}{c}
\dot{x}_{p, i} \\
\dot{z}_{p, i}
\end{array}\right]=\ldots+\kappa_{p, i} \sum_{j \neq i} \mathbf{R}\left(\boldsymbol{l}_{p, j} \theta_{i}^{j}\right)\left[\begin{array}{c}
\frac{\left(x_{p, j}-O_{p, j}\right)}{r_{p, j}} \\
\frac{z_{p, j}}{r_{p, j}}
\end{array}\right],
$$

where $p \in\{\mathrm{s}, \mathrm{f}\}$, represents the respective joint's unit-CPG. $i$ and $j$ represent the limb $\in \mathrm{LF}, \mathrm{RF}, \mathrm{LH}, \mathrm{RH}$.

The rotation matrix $\mathbf{R}\left(\theta_{i}^{j}\right)$ rotates the linear terms onto each other, where $\theta_{i}^{j}$ is the required relative phase between $i$ and $j$. Because we only use alternating gaits, the phase relationship $\theta_{i}^{j}$ can be calculated by specifying the gait phase $\varphi_{\mathrm{LH}}$, as presented in table I $\left(\theta_{i}^{j}=-\theta_{j}^{i}\right)$. Typically for trot $\varphi_{\mathrm{LH}}=0.5$, and for walk $\varphi_{\mathrm{LH}}=0.75$. Parameter $\kappa_{p, i}$ specifies

TABLE I

PHASE RELATIONSHIPS BETWEEN THE OSCILLATORS.

\begin{tabular}{||c|c|r||}
\hline \hline$i$ & $j$ & $\theta_{i}^{j}$ \\
\hline \hline LF & RF & $-\pi$ \\
\hline LF & LH & $-\varphi_{\mathrm{LH}} 2 \pi$ \\
\hline LF & RH & $\left(-\varphi_{\mathrm{LH}}+0.5\right) 2 \pi$ \\
\hline RF & LH & $\left(-\varphi_{\mathrm{LH}}+0.5\right) 2 \pi$ \\
\hline RF & RH & $\left(-\varphi_{\mathrm{LH}}+1\right) 2 \pi$ \\
\hline LH & RH & $\pi$ \\
\hline \hline
\end{tabular}

the coupling strengths into the unit-CPG $i$. The parameter $\imath_{p, j}$ is used to enable a correct coupling between unit-CPGs with different directions.

The coordination during the rhythmic movements is stable and flexible, and the generated trajectories are smooth, stable and robust to perturbations. The locomotor movements are easily modulated by the parameter set of each limb-CPG, the duty factor $\beta$ and gait phase $\varphi_{\mathrm{LH}}$.

This structure also allows omnidirectional locomotion because it is possible to independently control the step movements of the different joints and still maintain the intralimb coordination and interlimb coordination.



Fig. 2. Robot motion when performing a $\dot{\phi} \neq 0, v>0$ and $\phi_{\mathrm{w}}$. The robot walks in a circle path centered around the instantaneous center of rotation. The figure also depicts the orientation and length of the steps for achieving such walking motion.

\section{OMNIDIRECTIONAL LOCOMOTION}

The robot must be able to explore and navigate in its environment. For this reason it must be able to perform omnidirectional locomotion, i.e. to move to any point of interest, with a given translational speed, $v$, turning rate $\dot{\phi}$, and walking orientation $\phi_{\mathrm{w}}$ (fig. 2).

In order to achieve omnidirectional locomotion on our robotic platform, we take the idea from the wheel model presented in [12]. Basically, it assumes that each foot performs a step with a specified direction and length, and the overall propulsion of the steps results in the desired robot motion.

If feet movements are only in the robot sagittal plane, the robot moves straight. When feet movements are only in the robot transverse plane, it moves sideward. Movement in any direction is achieved by superimposing the movements of a foot on these two planes.

On the AIBO robot, the movements on the sagittal and transverse planes are carried by the hip swing and hip flap joints, respectively (fig. 1).

This method is well suited for this CPG network since both the step direction and length in the sagittal and transverse planes can be controlled independently for each leg.

To ensure the desired omnidirectional locomotion, described by the robot translation speed, the robot angular velocity and walking orientation; it is required to find the direction and amplitude of each hip swing and flap joints.

Because it is possible to describe the rotation of any point of the robot around a certain common point, where the translation speed is zero, i.e. the instantaneous centre of rotation (ICR), it is possible to find the trajectory of any point of the robot when performing such rotation. For the robot to move around a certain ICR, each limb should perform a step of proportional size into a suitable direction, tangential to the circle with radius $r=\frac{v_{\text {ref }}}{\dot{\phi}}$, around the rotation centre (fig. 2). By applying trigonometry, the amplitudes for hip swing $(s)$ and hip flap joints (f) are given as follows, according to the desired angular velocity, $\dot{\phi}$ and the desired walking 
orientation $\phi_{\mathrm{w}}$ :

$$
\begin{aligned}
A_{\mathrm{s}, i} & =A_{\mathrm{ref}} \frac{\dot{\phi} Y_{i}-v_{\mathrm{ref}} \cos \left(\phi_{\mathrm{w}}\right)}{v_{\mathrm{ref}}} . \\
A_{\mathrm{f}, i} & =-A_{\mathrm{ref}} \frac{\dot{\phi} X_{i}-v_{\mathrm{ref}} \sin \left(\phi_{\mathrm{w}}\right)}{v_{\mathrm{ref}}} .
\end{aligned}
$$

$\left(X_{i}, Y_{i}\right)$ are the limb $i$ coordinates in the robot reference frame. $v_{\text {ref }}$ is an approximate velocity value when using a certain amplitude $A_{\text {ref }}$ and a desired duty factor $\beta$. $A_{\text {ref }}$ is a maximum amplitude value set a priori, adequate for the robot.

\section{A. CPG modulation}

CPG parameters have to be set as needed to achieve the desired robot motion. For each of a limb's unit-CPG, a mechanism must determine their parameter values according to their roles in the final motion.

1) $v$, switches on/off the oscillatory behaviour;

2) $\mu$, modulates the amplitude of the solution;

3) $l$, sets the direction of the solution;

4) and $\beta$, relates with the robot's velocity by decreasing the duration of the stance phase.

1) Oscillatory behaviour: Qualitatively, by modifying on the fly the $v$ parameter, the system switches between a stable fixed point at $x=O$ (for $v=-1$ ) and a purely rhythmic movement (for $v=1$ ). The $v_{\mathrm{f}, i}$ and $v_{\mathrm{s}, i}$ parameters are set according to $A_{\mathrm{s}, i}$ and $A_{\mathrm{s}, i}$, respectively, as follows $(p=\{\mathrm{s}, \mathrm{f}\})$ :

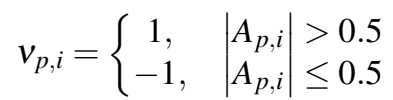

A dead zone is empirically set such that when the amplitude of the movement is negligible, the unit-CPG oscillatory behaviour is turned off.

For example, consider a situation in which the robot walks straight forward $\left(\phi_{w}=0^{\circ}\right)$ during $2.5 \mathrm{~s}$ and then a command of $\phi_{w}=90^{\circ}$ is given for the robot to propel sideways. Fig. 3 shows the two forelimbs trajectories. During the first $2.5 \mathrm{~s}$, the swing joints perform the locomotor movements, pushing the robot forward $\left(v_{\mathrm{s}, L F}, v_{\mathrm{s}, R F}=1\right)$. The flap joints do not move because they are not needed for propelling the robot straight forward $\left(v_{\mathrm{f}, L F}, v_{\mathrm{f}, R F}=-1\right)$. After $t=2.5 \mathrm{~s}$, the swing joints' rhythmic movements stop $\left(v_{\mathrm{s}, L F}, v_{\mathrm{s}, R F}=-1\right)$ and the flap joints start to move $\left(v_{\mathrm{f}, L F}, v_{\mathrm{f}, R F}=1\right)$.

2) Amplitude: Each unit-CPG's amplitude is modulated such that the corresponding controlled foot describes a step with the correct length. The $\mu$ parameter modulates the amplitude of each hip swing and flap unit-CPG, according to the $A_{\mathrm{s}, i}$ and $A_{\mathrm{f}, i}$ values, as follows $(p=\{\mathrm{s}, \mathrm{f}\})$ :

$$
\mu_{p, i}=A_{p, i}^{2}
$$

Fig. 4 shows the two forelimb joint movements when the robot is steering right $(\dot{\phi} \neq 0)$, while walking straight forward $\left(\phi_{w}=0^{\circ}\right)$. During the first $2 \mathrm{~s}, \dot{\phi}$ is set to $0.2 \mathrm{rad}^{-1}{ }^{-1}$. The outermost limbs (right) perform longer steps than the innermost limbs (left). At $t=2 \mathrm{~s}, \dot{\phi}$ is changed to -0.14 rad. $\mathrm{s}^{-1}$. The amplitudes change accordingly: the right limbs turn smaller than the left limb amplitudes.
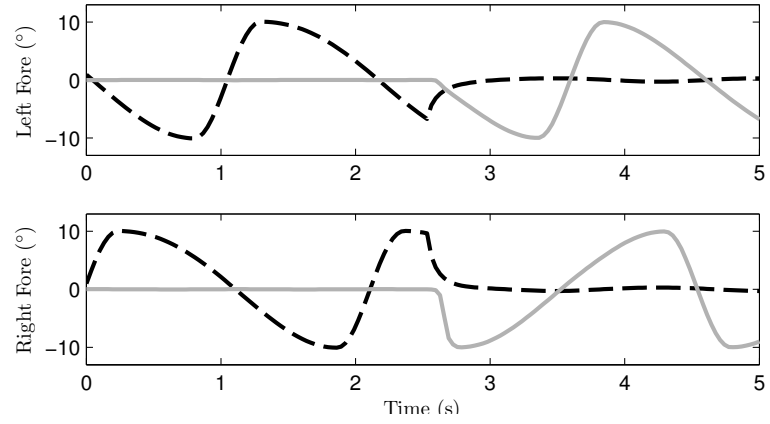

Fig. 3. Generated trajectories for the swing (dashed black) and flap (solid grey) joints in fore limbs. In the first half the robot walks forward, then at $2.5 \mathrm{~s}$ the rhythmic locomotor movements in the swing joints stop and start those of the flap joints, making the robot walk sideways.
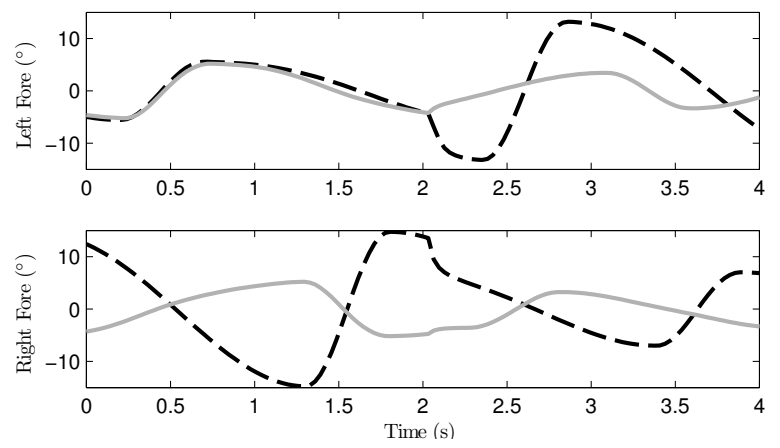

Fig. 4. Generated trajectories for the swing (dashed black) and flap (solid grey) joints in fore limbs. The robot steers to the left $\left(\dot{\phi}=0.2 \mathrm{rad}^{-1}\right)$ during the first $2 \mathrm{~s}$, and then to the right $\left(\dot{\phi}=-0.14 \mathrm{rad}_{\mathrm{s}} \mathrm{s}^{-1}\right)$. The amplitudes show that the steps on the outside of the robot are greater than those in the inside.

3) Step direction: The signs of $A_{\mathrm{s}, i}$ and $A_{\mathrm{f}, i}$ hold the information on the direction for the step movements of each unit-CPG.

Note that $l$ specifies the limit-cycle directions, i.e. counterclockwise or clockwise. Basically, changing the limit-cycle direction changes whether the step stance phase is ascending $(l=-1)$ or descending $(l=1)$ in the $x$ trajectory. Table II shows the assigned values of $\imath$.

TABLE II

$\imath$ VALUES FOR THE HIP SWING (S) AND HIP FLAP (F) UNIT-CPGS.

\begin{tabular}{||c|c|c|c|c||}
\hline \hline & $l_{\mathrm{s}, \mathrm{LF}}$ & $l_{\mathrm{s}, \mathrm{RF}}$ & $l_{\mathrm{s}, \mathrm{LH}}$ & $l_{\mathrm{s}, \mathrm{RH}}$ \\
\hline$A_{\mathrm{s}, i}>0$ & 1 & 1 & 1 & 1 \\
\hline$A_{\mathrm{s}, i}<0$ & -1 & -1 & -1 & -1 \\
\hline & $l_{\mathrm{f}, \mathrm{LF}}$ & $l_{\mathrm{f}, \mathrm{RF}}$ & $l_{\mathrm{f}, \mathrm{LH}}$ & $l_{\mathrm{f}, \mathrm{RH}}$ \\
\hline$A_{\mathrm{f}, i}>0$ & 1 & -1 & -1 & 1 \\
\hline$A_{\mathrm{f}, i}<0$ & -1 & 1 & 1 & -1 \\
\hline \hline
\end{tabular}

Results show that by online modifying the CPG parameters, we are able to modulate in real time the generated trajectories, and despite fast parameter changes the generated trajectories remain smooth.

\section{EXPERIMENTS}

Experiments were performed to validate the proposed method for generating the coordinated trajectories for om- 
nidirectional locomotion, as well as the CPG parameter modulation which encode movement description in terms of translational speed, angular velocity and walking orientation. Different experiments were set up to verify the adequacy of the CPG network both to omnidirectional movement generation and to verify if the resulting robot motion matches the specified high level commands. Experiments were performed both on Webots robotics simulator and on the AIBO robotic platform.

Many possible motions were tried over several runs by specifying different angular velocities and walking directions. Experiments were carried with a walking direction $\phi_{\mathrm{W}} \in[0,360]\left(^{\circ}\right)$ in steps of $5^{\circ}$, and angular velocity $\dot{\phi} \in$ $[0,0.5]\left(\mathrm{rad} . \mathrm{s}^{-1}\right)$ in steps of $0.03 \mathrm{rad} . \mathrm{s}^{-1}$. Experiments with the robot were carried on a flat environment with a grid of markers, spaced $20 \mathrm{~cm}$ apart. This grid enabled us to visually measure the performed path, comparing it with the expected path.

We only depict results obtained from three experiments with the real platform.

1) The robot only steers with a given angular velocity;

2) The robot walks diagonally;

3) The robot moves diagonally while steering with a given angular velocity.

We do not expect precise and exact motions, since the CPG approach is not intended for such a goal. We expect that the overall motion of the robot respects the specified motion commands within an acceptable but marginal error margin, specially because in this work the CPG based controller is open-loop and disregards physical effects and other disturbances, which should be used to improve the locomotion's performance.

\section{A. Steering}

We start by verifying the steering behavior, with a desired angular velocity. The robot walks forward, $\phi_{\mathrm{w}}=0^{\circ}$, performing a trot gait specified by $\beta=0.5$ and $\varphi_{\mathrm{LF}}=0.5$, with an angular velocity of $\dot{\phi}=-0.21 \mathrm{rad} . \mathrm{s}^{-1}$. For the given $\beta$ the approximate value for the robot velocity is $v_{\text {ref }} \approx 5.7 \mathrm{~cm} . \mathrm{s}^{-1}$. The robot is expected to perform a circle with a radius of $r_{\mathrm{ICR}} \approx 27 \mathrm{~cm}$. Fig. 5 presents an image composition of the experiment. It is possible to verify that the robot performs a full circle with an approximate radius of $25 \mathrm{~cm}$, a marginal error when compared to the overall setup dimensions. This error is both due to the physical effects, error measurements and the open loop nature of the proposed controller. However, despite the relevance of the last one, this was not one of the aims of the presented work and therefore should be disregarded.

\section{B. Diagonal walk}

In this experiment we verify the robot ability to walk with a given walking direction. A angular velocity $\dot{\phi}=0^{\circ}$ and a walking orientation $\phi_{\mathrm{w}}=-45^{\circ}$ are specified, meaning the robot is expected to walk diagonally, forward to the left, with equal forward and lateral velocities and no angular motion. It is expected that both the swing and flap joints perform

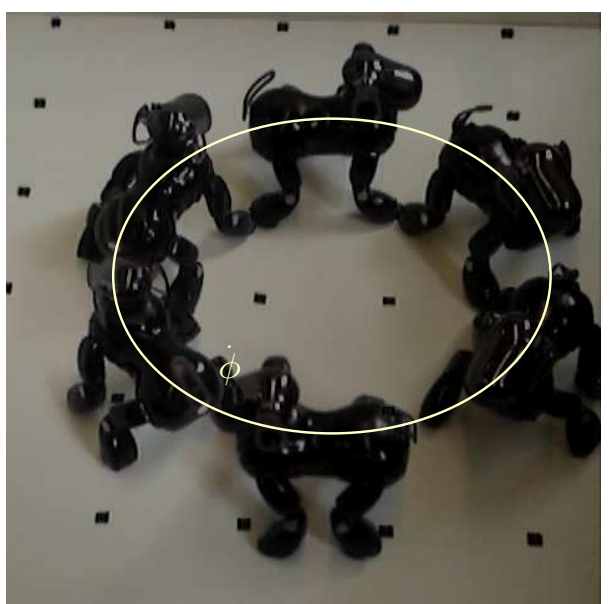

Fig. 5. The path described by the robot when $\dot{\phi}=-0.21$ rad. $\mathrm{s}^{-1}$ and $\phi_{\mathrm{w}}=0^{\circ}$. The resulting radius of the path is $\approx 25 \mathrm{~cm}$.

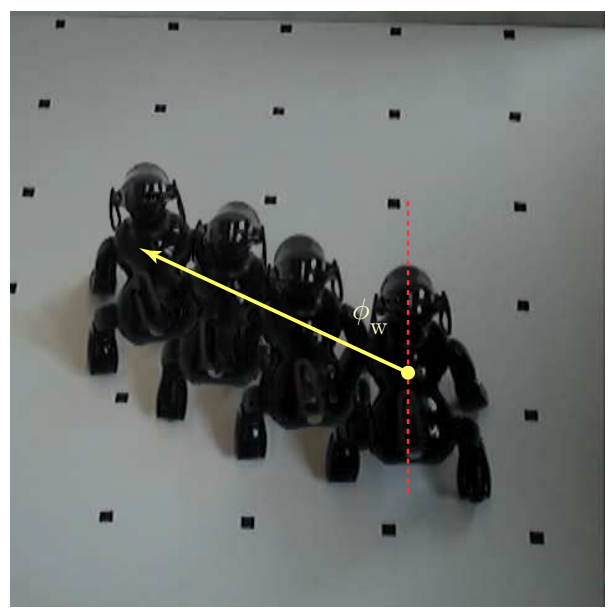

Fig. 6. The robot walks forward diagonally to the left, describing a path with $\dot{\phi}=0$ rad.s ${ }^{-1}$ and $\phi_{\mathrm{w}} \approx-45^{\circ}$.

stepping movements with the same amplitudes, propelling the robot equally forward and left. Fig. 6 shows the resulting robot behavior, a motion close to $-45^{\circ}$ diagonally.

\section{Steering diagonally}

If the robot moves with a certain walking orientation and angular velocity, it will perform a circular path while not heading straight forward, i.e. the body orientation will not be tangential to the circle specified by the angular velocity.

Again, the robot moves with a trot gait, specified by $\beta=0.5$ and $\varphi_{\mathrm{LF}}=0.5$, achieving a $v_{\mathrm{ref}} \approx 5.7 \mathrm{~cm} . \mathrm{s}^{-1}$. The walking orientation is set to $\phi_{\mathrm{w}}=65^{\circ}$ and angular velocity to $\dot{\phi}=0.27 \mathrm{rad} . \mathrm{s}^{-1}$. The robot is expected to walk while turning, heading about $\approx 65^{\circ}$ to the center of its circular path with $22 \mathrm{~cm}$ of radius. The obtained robot path is shown in fig. 7.

These experiments suggest that the calculation of the movement amplitudes and directions of each limb, despite being based on an approximate velocity value, can successfully be used to modulate the CPGs parameters in terms of the desired angular velocity and walking orientation. The 


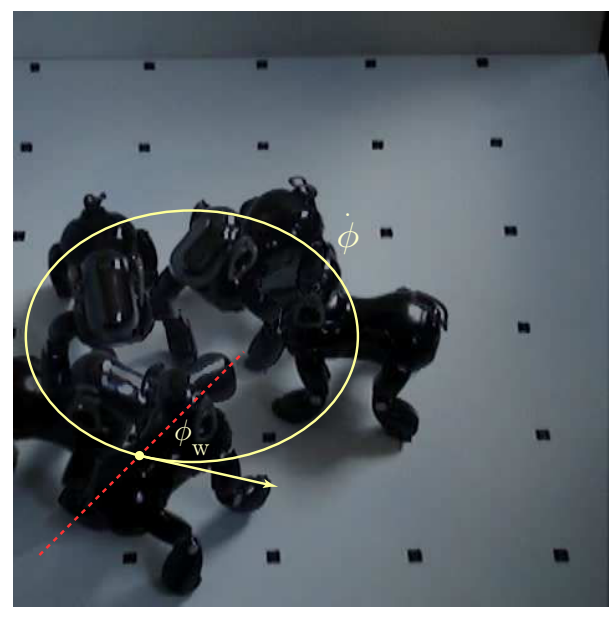

Fig. 7. The robot steers while heading the center of the path, with $\dot{\phi}=$ $0.27 \mathrm{rad} . \mathrm{s}^{-1}$ and $\phi_{\mathrm{w}}=-65^{\circ}$. It walks diagonally to the right while steering right, in a path with a radius of $\approx 22 \mathrm{~cm}$.

generated trajectories are modulated as required and the robot is able to perform omnidirectional locomotion.

In fact the obtained results have been quite satisfactory, especially considering the simplicity and open-loop nature of the system.

\section{CONCLUSIONS AND FUTURE WORKS}

This contribution is expected to be a step further in the more ambitious goal of proposing a bio-inspired architecture for goal-directed locomotion that outperforms current and existent solutions.

We proposed a CPG model and a CPG network capable of controlling the limb's joints in order to achieve omnidirectional quadruped locomotion. The proposed CPG was designed using the Hopf nonlinear oscillator, which presents several desirable advantages in robotic applications. Its application also allows the coordination of all the limbs in all the omnidirectional motions. The proposed CPG network achieves several quadruped alternating gaits, with independent step phase durations and possibility of performing steps in any direction.

One advantage of the proposed CPG model is the possibility of integrating sensory feedback mechanisms already presented in other works [8], [10] and the possibility to explore new ones.

In this work it was also presented a method for modulating all the CPG's parameters, requiring hand tuning for just a small number of parameters. The generated movements are modulated through higher level commands that encode the desired walking motion in terms of the translational speed, the walking orientation and the angular velocity; therefore reducing the dimensionality of the control problem.

In the end, experiment's results demonstrated that the proposed controller is capable of generating the required limb movements for omnidirectional locomotion.

\section{ACKNOWLEDGEMENTS}

Work supported by the Portuguese Science Foundation (grant PTDC/EEA-CRO/100655/2008).

\section{REFERENCES}

[1] F. Delcomyn, "Neural basis for rhythmic behaviour in animals," Science, no. 210, pp. 492-498, 1980.

[2] O. Kiehn, "Locomotor circuits in the mammalian spinal cord.," Annu Rev Neurosci, vol. 29, pp. 279-306, 2006.

[3] E. Bizzi, A. dAvella, P. Saltiel, and M. Trensch, "Modular organization of spinal motor systems," The Neuroscientist, no. 8, p. 2002, 2002.

[4] G. Schner and M. Dose, "A dynamical systems approach to tasklevel system integration used to plan and control autonomous vehicle motion.," Robotics and Autonomous Systems, vol. 10, no. 4, pp. 253 267, 1992.

[5] S. Schaal, S. Kotosaka, and D. Sternad, "Nonlinear dynamical systems as movement primitives, presented at," in First IEEE/RAS International Conference on Humaniod Robotics (Humanoids 2000, 2000.

[6] A. J. Ijspeert, J. Nakanishi, and S. Schaal, "Learning attractor landscapes for learning motor primitives," in Advances in Neural Information Processing Systems 15, pp. 1547-1554, MIT Press, 2002.

[7] S. Degallier, L. Righetti, and A. Ijspeert, "Hand placement during quadruped locomotion in a humanoid robot: A dynamical system approach," in IEEE-RAS International Conference on Intelligent Robots and Systems (IROSO7), 2007.

[8] L. Castro, C. Santos, M. Oliveira, and A. Ijspeert, "Postural control on a quadruped robot using lateral tilt: A dynamical system approach.," in EUROS, vol. 44 of Springer Tracts in Advanced Robotics, pp. 205214, Springer, 2008.

[9] S. Degallier, C. Santos, L. Righetti, and A. Ijspeert, "Movement generation using dynamical systems: a humanoid robot performing a drumming task," in IEEE-RAS International Conference on Humanoid Robots, 2006.

[10] L. Righetti and A. J. Ijspeert, "Pattern generators with sensory feedback for the control of quadruped locomotion," in 2008 IEEE International Conference on Robotics and Automation, 2008.

[11] V. Matos, C. Santos, and C. Pinto, "A brainstem-like modulation approach for gait transition in a quadruped robot," in 2009 IEEE IROS, 2009.

[12] B. Hengst, D. Ibbotson, S. B. Pham, and C. Sammut, "Omnidirectional locomotion for quadruped robots," in RoboCup 2001: Robot Soccer World Cup V, (London, UK), pp. 368-373, Springer-Verlag, 2002.

[13] H. Kimura, Y. Fukuoka, and A. H. Cohen, "Adaptive dynamic walking of a quadruped robot on natural ground based on biological concepts," Int. Journal of Robotics Research, vol. 26, no. 5, pp. 475-490, 2007.

[14] A. Rizzi and D. Koditschek, "Further progress in robot juggling: Solvable mirror laws," in IEEE International Conference on Robotics and Automation, pp. 2935-2940, 1994.

[15] T. FUKUDA, Y. ADACHI, H. HOSHINO, E. MURO, and K. KURASHIGE, "Omnidirectional walking mechanism : Redundancy and trajectory control," JSME international journal. Series $C$, Mechanical systems, machine elements and manufacturing, vol. 40, no. 4, pp. 694-701, 19971215.

[16] L. Zhang, S. Ma, and K. Inoue, "Several insights into omnidirectional static walking of a quadruped robot on a slope," in IROS, pp. 52495254, 2006.

[17] X. Chen, K. Watanabe, K. Kiguchi, and K. Izumi, "Implementation of omnidirectional crawl for a quadruped robot," Advanced Robotics, vol. 15, no. 2, pp. 169-190, 2001.

[18] Z. J. Kolter and A. Ng, "Learning omnidirectional path following using dimensionality reduction," in Proceedings of Robotics: Science and Systems, (Atlanta, GA, USA), June 2007.

[19] K. Tsujita, H. Toui, and K. Tsuchiya, "Dynamic turning control of a quadruped locomotion robot using oscillators," Advanced Robotics, vol. 19, pp. 1115-1133(19), October 2005.

[20] H. Kimura, Y. Fukuoka, and H. Katabuti, "Mechanical design of a quadruped "tekken3 \& 4" and navigation system using laser range sensor," in Proceedings of International Symphosium of Robotics, (Tokyo), November 2005. 